\title{
Magnetodielectric effect in EuZrO3
}

$\operatorname{AUTHOR}(S)$ :

Kolodiazhnyi, T.; Fujita, K.; Wang, L.; Zong, Y.; Tanaka, K.; Sakka, Y.; Takayama-Muromachi, E.

CITATION:

Kolodiazhnyi, T. ... [et al]. Magnetodielectric effect in EuZrO3. APPLIED PHYSICS LETTERS 2010, 96(25): 252901.

\section{ISSUE DATE:}

2010-06

URL:

http://hdl.handle.net/2433/147190

\section{RIGHT:}

Copyright 2010 American Institute of Physics. This article may be downloaded for personal use only. Any other use requires prior permission of the author and the American Institute of Physics. The following article appeared in APPLIED PHYSICS LETTERS96, 252901 (2010) and may be found at http://link.aip.org/link/APPLAB/v97/i16/p163503_s1 


\title{
Magnetodielectric effect in $\mathrm{EuZrO}_{3}$
}

\author{
T. Kolodiazhnyi, ${ }^{1, a)}$ K. Fujita, ${ }^{2}$ L. Wang, ${ }^{3}$ Y. Zong, ${ }^{2}$ K. Tanaka, ${ }^{2}$ Y. Sakka, ${ }^{3}$ and \\ E. Takayama-Muromachi ${ }^{1}$ \\ ${ }^{1}$ National Institute for Materials Science, 1-1 Namiki, Tsukuba, Ibaraki 305-0044, Japan \\ ${ }^{2}$ Department of Material Chemistry, Graduate School of Engineering, Kyoto University, Katsura, Nishikyo, \\ Kyoto 615-8510, Japan \\ ${ }^{3}$ Graduate School of Pure and Applied Sciences, University of Tsukuba, 1-1-1 Tenodai, Tsukuba, \\ Ibaraki 305-8571, Japan and National Institute for Materials Science, 1-2-1 Sengen, Tsukuba, \\ Ibaraki 305-0047, Japan
}

(Received 25 April 2010; accepted 3 June 2010; published online 21 June 2010)

\begin{abstract}
Following recent report on antiferromagnetic ordering in $\mathrm{EuZrO}_{3}$ we performed dielectric measurements of this material as a function of temperature and magnetic field. Dielectric constant of dense $\mathrm{EuZrO}_{3}$ ceramics is 30.1 at $300 \mathrm{~K}$. It gradually decreases upon cooling without any quantum paraelectric behavior; however, below $T_{\mathrm{N}}=4.1 \mathrm{~K}$ it shows a pronounced drop that qualitatively resembles that observed in $\mathrm{EuTiO}_{3}$. We report that dielectric constant of $\mathrm{EuZrO}_{3}$ is magnetic field dependent. The magnitude of the magnetodielectric effect in both $\mathrm{EuTiO}_{3}$ and $\mathrm{EuZrO}_{3}$ is discussed in the light of the recently proposed coupling of the Eu-O-Eu superexchange interactions with electrons involved in partially covalent $\mathrm{Ti}(\mathrm{Zr})-\mathrm{O}$ bond. () 2010 American Institute of Physics. [doi:10.1063/1.3456730]
\end{abstract}

Recent interest in magnetoelectric materials has brought to light several compounds containing divalent europium. For example, $\mathrm{EuTiO}_{3}$, a G-type antiferromagnet below Néel temperature, $T_{\mathrm{N}}=5.5 \mathrm{~K}$, demonstrates a strong magnetocapacitance effect. ${ }^{1,2}$ In view of very low electrical conductivity of $\mathrm{EuTiO}_{3}$ at $T \leq 60 \mathrm{~K}$, observed magnetocapacitance is attributed solely to the magnetic field dependence of dielectric constant, $\varepsilon(H)$. Most recently, electric field induced magnetoelectric coupling in $\mathrm{EuTiO}_{3}$ has been reported by Shvartsman $e t a l .^{3}$

Although theoretical support for magnetoelectric coupling in $\mathrm{EuTiO}_{3}$ is abundant, ${ }^{4}$ a direct proof of magnetic dependence of the optical phonon mode(s) has yet to be found. Recent analysis of the infrared active phonon modes in $\mathrm{EuTiO}_{3}$ indicates that, similar to $\mathrm{SrTiO}_{3}$, the (soft) phonon mode that contributes the largest dielectric weight to the phonon spectrum and presumably is responsible for the magnetoelectric effect, is Slater-type mode. ${ }^{5}$ Remarkably, this mode does not involve magnetically active $\left(\mathrm{Eu}^{2+}\right)$ ions, as opposed to the Last-type mode where $\mathrm{Eu}^{2+}$ ions vibrate against the $\mathrm{TiO}_{6}$ octahedra. According to Cohen, ${ }^{6}$ the properties of the Slater-type mode in the $\mathrm{A} \mathrm{O}_{3}$ perovskites with $\mathrm{A}=\mathrm{Sr}, \mathrm{Ba}$, and $\mathrm{Pb}$ and $B=\mathrm{Ti}$ are largely dependent on the overlap between the $\mathrm{O} 2 p$ and $B 3 d$ orbitals, i.e., on the degree of covalency of the $B-\mathrm{O}$ bond. Recently, Goian et al., ${ }^{5}$ have suggested that the magnetoelectric effect in $\mathrm{EuTiO}_{3}$ can be understood in terms of the impact of Eu-O-Eu superexchange on the electron density involved in (partially) covalent $\mathrm{Ti}-\mathrm{O}$ bonding. One way to examine this hypothesis is to find another $\mathrm{Eu}^{2+} B^{4+} \mathrm{O}_{3}$ perovskite with similar magnetic structure but different degree of covalency of the $B-\mathrm{O}$ bond.

Here we report on synthesis, crystal structure, and physical properties of $\mathrm{EuZrO}_{3}$ perovskite with a special emphasis on its magnetodielectric effect. Until recently, $\mathrm{EuZrO}_{3}$ was considered paramagnetic with a cubic (space group $P m \overline{3} m$ )

${ }^{a)}$ Electronic mail: kolodiazhnyi.taras@nims.go.jp. perovskite structure. ${ }^{7}$ Recently Viallet et al. ${ }^{8}$ and Zong et al. ${ }^{9}$ have revised the crystal structure of $\mathrm{EuZrO}_{3}$ and found that it crystallizes in orthorhombic (space group Pbnm) symmetry similar to that of $\mathrm{SrZrO}_{3}$. Most importantly, Zong et al. ${ }^{9}$ found that $\mathrm{EuZrO}_{3}$ develops an antiferromagnetic (AFM) ordering below $T_{\mathrm{N}} \approx 4.1 \mathrm{~K}$. Although direct confirmation of the type of the AFM structure in $\mathrm{EuZrO}_{3}$ has yet to be provided by neutron diffraction, the indirect evidence indicates that $\mathrm{EuZrO}_{3}$ and $\mathrm{EuTiO}_{3}$ are magnetically very similar. In particular, analysis of the sign and magnitude of the magnetic exchange constants, $J_{i}$, between nearest, $J_{1}$, and nextnearest, $J_{2}, \mathrm{Eu}^{2+}$ ions in $\mathrm{EuZrO}_{3}$ (Ref. 9) favors the G-type AFM structure, similar to that found in $\mathrm{EuTiO}_{3} \cdot{ }^{10,11}$ On the other hand, one may expect the valence electronic structure of these two perovskites to be quite different. Namely, in contrast to partially covalent $\mathrm{Ti}-\mathrm{O}$ bond in $\mathrm{EuTiO}_{3}$, the $\mathrm{Zr}-\mathrm{O}$ bond in $\mathrm{EuZrO}_{3}$ is more ionic, similar to the case of $\mathrm{SrZrO}_{3}$. This makes $\mathrm{EuZrO}_{3}$ an important reference material for understanding of the magnetodielectric effect in $\mathrm{Eu}^{2+}$-based perovskites.

EuZrO${ }_{3}$ was prepared from $\mathrm{Eu}_{2} \mathrm{O}_{3}$ and $\mathrm{ZrO}_{2}$ (both $99.9 \%$ pure, sourced from Kanto Chemicals, Japan). The stoichiometric mixture of $\mathrm{Eu}_{2} \mathrm{O}_{3}$ and $\mathrm{ZrO}_{2}$ was treated at $1300{ }^{\circ} \mathrm{C}$ for $20 \mathrm{~h}$ in pure hydrogen at a flow rate of $80 \mathrm{~cm}^{3} / \mathrm{min}$, with intermediate regrinding until a yellow, single phase $\mathrm{EuZrO}_{3}$ product was obtained. Phase purity was confirmed by powder x-ray diffraction (Rigaku Ultima III x-ray diffractometer, $\lambda=0.15406 \mathrm{~nm}$ radiation). Lattice parameters were obtained from Rietveld refinement of the x-ray data using RIETAN 2000. Magnetic susceptibility in the $2-100 \mathrm{~K}$ range was measured using superconducting quantum interference devise (Quantum Design, MPMS, USA). $\mathrm{EuZrO}_{3}$ ceramic with $98 \%$ density was obtained by spark plasma sintering (SPS) (Dr. Sinter, SPS-1050, Japan) at $1450{ }^{\circ} \mathrm{C}$ under 130 $\mathrm{MPa}$ uniaxial pressure and base vacuum pressure of $2 \times 10^{-2} \mathrm{~Pa}^{12}$ To release the residual lattice strain after SPS, the samples were annealed at $1300{ }^{\circ} \mathrm{C}$ in $\mathrm{H}_{2}$ gas for $20 \mathrm{~h}$. For dielectric measurements, Au electrodes were spattered 


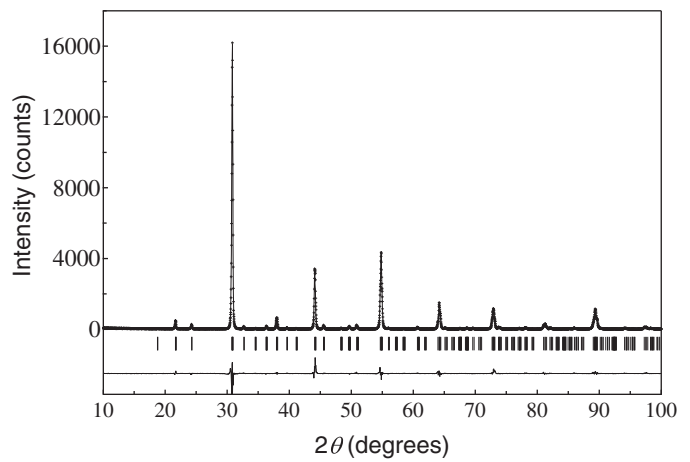

FIG. 1. Room temperature $\mathrm{x}$-ray diffraction pattern of $\mathrm{EuZrO}_{3}(+)$. Calculated diffraction pattern from Rietveld refinement of $\mathrm{EuZrO}_{3}$ (solid line). The vertical bars indicate the positions of expected Bragg peaks. The difference between observed and calculated data is shown at the bottom of the plot.

on the $\mathrm{EuZrO}_{3}$ disks of $7 \mathrm{~mm}$ diameter and $1 \mathrm{~mm}$ thickness. Dielectric properties were measured with Agilent E4980 Precision LCR Meter in the temperature interval of 2.5-300 K and magnetic field of 0-80 kOe utilizing a home-made dielectric measurements probe coupled with the Physical Property Measurement System (QD, USA).

Powder X-ray analysis (Fig. 1) has confirmed orthorhombic (space group Pbnm) symmetry of $\mathrm{EuZrO}_{3}$ with lattice constants $a=5.8019(4), b=5.8236(5)$, and $c=8.2051(9) \AA$. The x-ray pattern was refined to the reliability factor $R_{\mathrm{p}}$ $=11.2 \%$ in close agreement with Ref. 8. According to the lattice parameters, the absolute density of $\mathrm{EuZrO}_{3}$ is $6.976 \mathrm{~g} \mathrm{~cm}^{-3}$

Temperature dependence of magnetic susceptibility, $\chi$, measured at $H=50$ Oe and shown in Fig. 2 indicates an AFM phase transition at $T_{\mathrm{N}} \approx 4.1 \mathrm{~K}$. Linear fit of the $\chi^{-1}(T)$ dependence yields a Weiss temperature $\theta_{W} \approx+0.1 \mathrm{~K}$. According to Ref. 9, positive $\theta_{W}$ is a rare case that indicates coexistence of both ferromagnetic and AFM interactions in $\mathrm{EuZrO}_{3}$. Remarkably, positive $\theta_{W} \approx+3.3 \mathrm{~K}$ has also been found in $\mathrm{EuTiO}_{3}$, thus supporting similar G-type AFM magnetic structure.

Since $\mathrm{EuZrO}_{3}$ and $\mathrm{SrZrO}_{3}$ are structurally identical with the difference in the lattice constants of $\approx 0.04 \%,{ }^{13}$ one may expect similar dielectric properties. Indeed, room tempera-

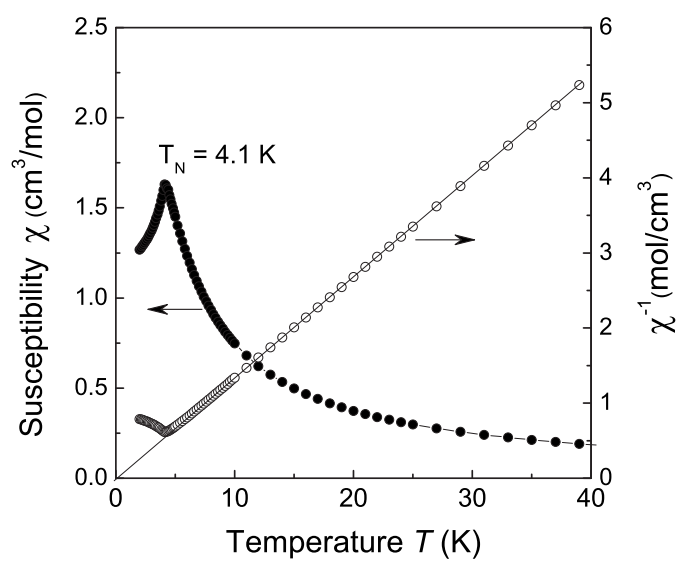

FIG. 2. Temperature dependence of zero field cooled molar magnetic susceptibility, $\chi$, and $\chi^{-1}$ of $\mathrm{EuZrO}_{3}$. The AFM transition at $T_{\mathrm{N}} \approx 4.1 \mathrm{~K}$ is detected from the $\chi(T)$ anomaly. Linear fit of the $\chi^{-1}(T)$ dependence yields a positive Weiss temperature, $\theta_{W} \approx+0.1 \mathrm{~K}$.

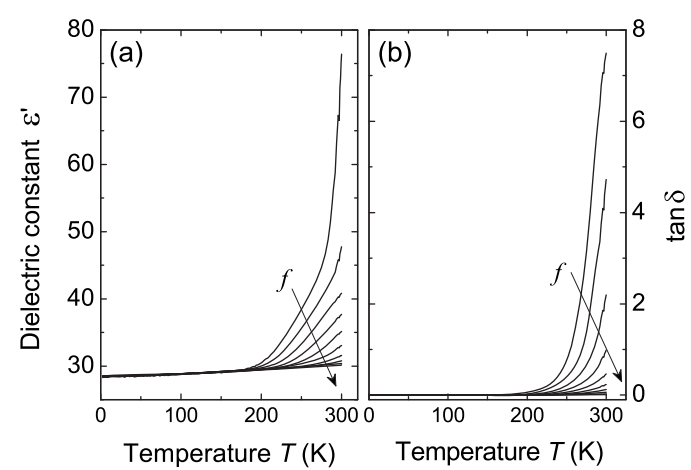

FIG. 3. Temperature dependence of (a) dielectric constant, $\varepsilon^{\prime}$ and (b) dielectric loss, $\tan \delta$, of $\mathrm{EuZrO}_{3}$ at selected frequencies $f=0.1,0.3,0.7,1.7$, $4.4,11.3,29.2,75.3,194$, and $500 \mathrm{kHz}$.

ture $\varepsilon^{\prime}$ of $\mathrm{EuZrO}_{3}$ determined in this work and that of $\mathrm{SrZrO}_{3}$ reported in Ref. 13 are 30.1 and 30.4, respectively. Dielectric constant of $\mathrm{EuZrO}_{3}$ is weakly temperature dependent [Fig. 3(a)] with $d \varepsilon^{\prime} / d T \approx 6.8 \times 10^{-3} \mathrm{~K}^{-1}$. The $\varepsilon^{\prime}$ gradually decreases from 30.1 at $300 \mathrm{~K}$ to 28.45 at $5 \mathrm{~K}$ with no indication of the quantum paraelectric behavior. This supports our original assumption of the predominantly ionic $\mathrm{Zr}-\mathrm{O}$ bond which does not favor formation of the lowenergy (soft) phonon mode in $\mathrm{EuZrO}_{3}$. Above $175 \mathrm{~K}, \varepsilon^{\prime}$ shows low-frequency dispersion which, we believe, is attributed to the finite electronic conductivity also evidenced from the large $\tan \delta$ values [Fig. 3(b)]. From the linear $\tan \delta$ vs. $\omega^{-1}$ dependence (not shown) we conclude that above $175 \mathrm{~K}$ the imaginary part of the dielectric constant is dominated by the electronic conductivity, $\sigma^{\prime}$, according to

$$
\varepsilon^{*}=\varepsilon^{\prime}+i \frac{\sigma^{\prime}}{\varepsilon_{0} \omega},
$$

where $\varepsilon_{0}$ is the electric constant and $\omega$ is the angular frequency, $\omega=2 \pi f$.

The low-temperature behavior of $\varepsilon^{\prime}$ is shown in Fig. 4(a) as a function of applied magnetic field. Dielectric constant of $\mathrm{EuZrO}_{3}$ at $H=0$ demonstrates an anomalous drop below $T$ $\approx 4 \mathrm{~K}$, the temperature corresponding to the AFM phase transition. Pronounced change in the $\varepsilon^{\prime}(T)$ dependence is found upon application of magnetic field. Low- $T$ dielectric constant increases with magnetic field and saturates at $\varepsilon^{\prime}$ $\approx 28.48$ for $H \geq 30 \mathrm{kOe}$. Qualitatively, magnetodielectric effect in $\mathrm{EuZrO}_{3}$ is similar to that reported for $\mathrm{EuTiO}_{3}$. On the quantitative scale, the magnitude of the magnetodielectric effects in $\mathrm{EuZrO}_{3}$ and $\mathrm{EuTiO}_{3}$ is quite different. Katsufuji et al. ${ }^{1}$ proposed a simple equation to describe the magnetodielectric effect as follows:

$$
\varepsilon^{\prime}(H)=\varepsilon^{\prime}(H=0)\left(1+\alpha\left\langle\mathbf{S}_{i} \cdot \mathbf{S}_{j}\right\rangle\right),
$$

where $\varepsilon^{\prime}(H=0)$ is dielectric constant at zero magnetic field, $\left\langle\mathbf{S}_{i} \cdot \mathbf{S}_{j}\right\rangle$ is the spin pair correlation between nearest neighbor $\mathrm{Eu}^{2+}$, and $\alpha$ is the coupling constant between spin correlation and dielectric constant. In order to estimate $\left\langle\mathbf{S}_{i} \cdot \mathbf{S}_{j}\right\rangle$ in $\mathrm{EuZrO}_{3}$, we have performed a mean-field calculation based on a Heisenberg model under an assumption that the $4 f$ spins of $\mathrm{Eu}^{2+}(\mathrm{S}=7 / 2)$ are located on a simple cubic lattice with a G-type AFM ordering. ${ }^{9}$ The Heisenberg Hamiltonian of the spin system, with restriction to the nearest-neighbor and next-nearest-neighbor interactions, is given by 


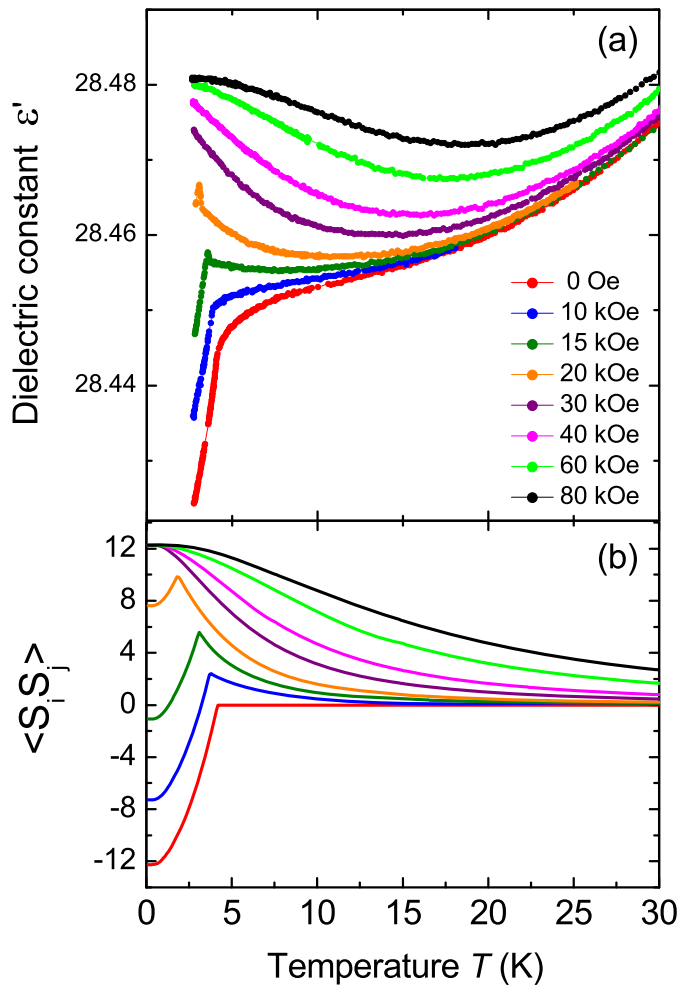

FIG. 4. (Color online) (a) Low-temperature behavior of dielectric constant of EuZrO $\mathrm{Zr}_{3}$ measured at $200 \mathrm{kHz}$ in magnetic field of 0,10,15, 20, 30, 40, 60 , and $80 \mathrm{kOe}$. (b) Spin correlation function for cubic EuZrO 3 calculated using a mean-field approximation.

$$
H=-2 J_{1} \sum_{\langle i, j\rangle} \mathbf{S}_{i} \cdot \mathbf{S}_{j}-2 J_{2} \sum_{\{i, j\}} \mathbf{S}_{i} \cdot \mathbf{S}_{j}
$$

where $\mathbf{S}_{i}$ is the operator of the $4 f$ spin localized at the site $i$. The sums of the first and the second terms run over the nearest-neighbor $\langle i, j\rangle$, and the next-nearest-neighbor pairs $\{i, j\}$, respectively. In our calculation, we used $J_{1} / k_{B}=-0.032 \mathrm{~K}$ and $J_{2} / k_{B}=0.017 \mathrm{~K}$ which yield $T_{\mathrm{N}}$ $=4.1 \mathrm{~K}$ and $\theta_{W}=0.1 \mathrm{~K}$, where $k_{B}$ is the Boltzmann constant. Figure 4(b) shows temperature dependence of $\left\langle\mathbf{S}_{i} \cdot \mathbf{S}_{j}\right\rangle$ calcu- lated from the product of $\langle S\rangle$ on the two sublattices under different $H$.

From the data given in Figs. 4(a) and 4(b) and using Eq. (2), one finds for EuZrO ${ }_{3}$ the magnetodielectric coupling constant $\alpha \approx 1.1 \times 10^{-4}$, which is a factor of 27 lower than that of $\mathrm{EuTiO}_{3}$ (i.e., $\alpha \approx 2.74 \times 10^{-3}$ ). ${ }^{1}$

In conclusion, at low $T, \mathrm{EuZrO}_{3}$ shows magnetodielectric effect whose features are qualitatively similar to that reported for $\mathrm{EuTiO}_{3}$, albeit the former perovskite is not a quantum paraelectric. That said, the magnitude of the magnetodielectric coupling in $\mathrm{EuZrO}_{3}$ is much smaller than that of $\mathrm{EuTiO}_{3}$. In accord with the hypothesis of Goian et al., ${ }^{5}$ we attribute this difference to stronger covalency of the Ti-O bond in $\mathrm{EuTiO}_{3}$ which favors formation of the soft phonon mode which, in turn, facilitates coupling with the low-energy magnetic excitations.

This work has been supported by Grant-in-Aid for Scientific Research Grant No. 21560025 (T.K.) and Scientific Research on Priority Area Grant No. 22015011 (K.F.) from MEXT Japan.

${ }^{1}$ T. Katsufuji and H. Takagi, Phys. Rev. B 64, 054415 (2001).

${ }^{2}$ S. Kamba, D. Nuzhnyy, P. Vanĕk, M. Savinov, K. Knízek, Z. Shen, E. Šantavá, K. Maca, M. Sadowski, and J. Petzelt, EPL 80, 27002 (2007).

${ }^{3}$ V. V. Shvartsman, P. Borisov, W. Kleemann, S. Kamba, and T. Katsufuji, Phys. Rev. B 81, 064426 (2010).

${ }^{4}$ H. Wu, Q. Jiang, and W. Z. Shen, Phys. Rev. B 69, 014104 (2004); R. Ranjan, H. S. Nabi, and R. Pentcheva, J. Phys.: Condens. Matter 19 , 406217 (2007).

${ }^{5}$ V. Goian, S. Kamba, J. Hlinka, P. Vanĕk, A. A. Belik, T. Kolodiazhnyi, and J. Petzelt, Eur. Phys. J. B 71, 429 (2009).

${ }^{6}$ R. E. Cohen, Nature (London) 358, 136 (1992).

${ }^{7}$ A. H. Shafer, J. Appl. Phys. 36, 1145 (1965).

${ }^{8}$ V. Viallet, J.-F. Marucco, J. Saint, M. Herbst-Ghysel, and N. Dragoe, J. Alloys Compd. 461, 346 (2008).

${ }^{9}$ Y. Zong, K. Fujita, H. Akamatsu, S. Murai, and K. Tanaka, J. Solid State Chem. 183, 168 (2010).

${ }^{10}$ T. R. McGuire, M. W. Shafer, R. J. Joenk, H. A. Alperin, and S. J. Pickart, J. Appl. Phys. 37, 981 (1966).

${ }^{11}$ J. E. Greedan, C. L. Chien, and R. G. Johnston, J. Solid State Chem. 19, 155 (1976).

${ }^{12}$ S. Grasso, Y. Sakka, and G. Maizza, Sci. Technol. Adv. Mater. 10, 053001 (2009).

${ }^{13}$ D. Souptel, G. Behr, and A. M. Balbashov, J. Cryst. Growth 236, 583 (2002). 